\title{
EFFECTS OF CHANGING ATTENTIONAL FOCUS REMINDER RATES ON LEARNING TO THROW DARTS
}

\author{
A Thesis \\ presented to \\ the Faculty of California Polytechnic State University, \\ San Luis Obispo, California
}

In Partial Fulfillment

of the Requirements for the Degree

Master of Science in Kinesiology

By

Arya Alami

September 2010 
(C) 2010

Arya Alami

ALL RIGHTS RESERVED

ii 


\section{COMMITTEE MEMBERSHIP}

TITLE:

AUTHOR:

DATE SUBMITTED:

COMMITTEE CHAIR:

COMMITEE MEMBER: Camille P. O'Bryant, PhD

COMMITTE MEMBER: Suzanne Phelan, PhD
Effects of Changing Attentional Focus Reminder Rates on Learning to Throw Darts

Arya Alami

September 2010

Kellie Green Hall, PhD 


\begin{abstract}
Effects of Changing Attentional Focus Reminder Rates on Learning to Throw Darts
\end{abstract} Arya Alami

The purpose of the study was to examine the effects of an external focus of attention on the learning of dart throwing at three different focus reminder frequencies (every two, every four and every ten trials). Twenty-four male and female subjects were randomly assigned to one of three groups. Subjects threw darts at a circular target 60 times over two days while getting a reminder of their intended external focus at the three different reminder frequencies. Five-day delayed retention and transfer tests were conducted to assess learning, each consisting of five trials. The target was comprised of five concentric circles, with the center zone worth five points and the outer-most zone worth one point. Target scores were used for statistical analysis. Findings show that the groups given a reminder after every fourth and tenth trial perform better during acquisition $(\mathrm{F}=13.61 ; \mathrm{p}<0.001)$. Furthermore, the group that received a reminder after every tenth trial performed the best during the retention test. Although, the high variability within groups may have prevented more significant differences between reminder frequency groups in the retention and transfer test results, it is evident that lessfrequent reminders result in better performance and learning of dart throwing. 
Table of Contents

Page

List of Tables and Figures........................................ vii

Chapter

1. Introduction................................................. 1

Background of Study $\ldots \ldots \ldots \ldots \ldots \ldots \ldots \ldots \ldots \ldots \ldots \ldots \ldots \ldots \ldots \ldots$

Statement of the Problem..................................... 3

Hypothesis........................................... 3

Delimitations......................................... 3

Assumptions......................................... 3

Limitations.......................................... 4

Definition of Terms.................................... 4

2. Review of Literature........................................ 5

Summary............................................... 26

3. Methods and Procedures.................................... 28

Restatement of the Purpose............................... 28

Subjects.......................................... 28

Apparatus and Task.................................... 28

Procedures............................................... 30

Data Analysis........................................ 32

4. Results................................................... 33

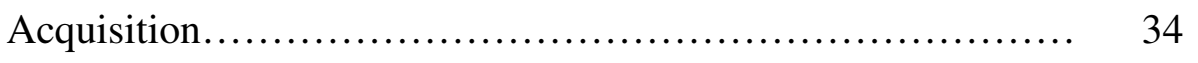

Retention............................................... 35 
Transfer.............................................. 36

5. Discussion.................................................... 38

Recommendations...................................... 40

Conclusion................................................ 40

References............................................... 42

Appendices................................................. 45

A. Statistical Output.................................... 45

Acquisition Results............................. $\quad 45$

Retention Test Results............................. 46

Transfer Test Results............................. 47

B. Informed Consent..................................... 48 
List of Tables and Figures

Tables $\quad$ Page

1. Summary of Attentional Focus Studies....................... 27

2. Acquisition Trial Blocks Scores \& Standard Deviation............. 34

3. Transfer Test Mean Scores \& Standard Deviation.................. 37

Figures

1. Wall with Corkboard and Dartboard........................... 29

2. Acquisition Blocks with Trendlines........................ 35

3. Acquisition \& Retention and Transfer Tests.................... 36 


\section{Chapter 1}

Introduction

\section{Background of the Study}

Success in learning can be measured by levels of proficiency. More often than not, the sooner one learns a new motor skill, the better. This fundamental idea holds true whether the task is learned in a classroom, on a sports field or in a rehabilitation center. Many tools, both physical and instructional, have been used to facilitate and expedite the learning process. One effective instructional tool is the use of attentional focus strategies. Using attentional focus strategies requires individuals to focus their attention on an aspect of either the movement (an internal focus) or the effects of the movement (an external focus) when performing a motor skill (Magill, 2007). The majority of the current research on attentional focus has found that the use of an external focus leads to better learning when practicing a novel skill (Wulf \& Weigelt, 1997, Shea \& Wulf, 1999, McNevin, Shea \& Wulf, 2003, Zachry, Wulf, Mercer \& Bezodis, 2005, etc). While there has been a consensus as to which type of focus leads to better learning, an optimal frequency at which participants should be reminded of their intended focus has yet to be established.

In every study examining the effects of attentional focus strategies, the participants received verbal reminders on their intended focus cue. However, none of these studies have come to a consensus as to how often a focus cue reminder should be given. The amount and frequency of reminders can affect learning. Too few reminders can result in participants losing focus on the intended focus cue and focusing on anything they deem important. Too many reminders, however, may interfere with processing, 
similar to a guidance hypothesis effect (Schmidt \& Lee, 2005). Although guidance has been shown to improve performance during trials in which it is administered, researchers have found that it prevents proper learning from taking place by allowing the learner to rely on the given statement or feedback (Salmoni, Schmidt \& Walter, 1984). In previous literature, the frequency of reminders has ranged from every trial to every ten trials. Typically, researchers choose to give focus cue reminders at the end of each trial block. Within the past ten years, attentional focus research has garnered a lot of attention. Researchers have attempted to better describe the differences between an external and an internal focus, using many different novel skills and tasks. Currently, studies conducted on motor skills, such as a golf swing (Wulf, Lauterbach \& Toole, 1999), a tennis stroke (Wulf, McNevin, Fuchs, Ritter \& Toole, 2000), a basketball free throw shot (Zachry, Wulf, Mercer \& Bezodis, 2005) and dart throwing (Radlo, Steinberg, Singer, Barba \& Melnikov, 2002) all show the advantageous effects of an external focus on learning a new skill. However, none of the aforementioned researchers used the same reminder frequency nor do they explain the decision of the reminder frequency used.

While previous authors have suggested that an external focus of attention leads to better performance and learning, there is a need for a universally accepted frequency at which reminders of attentional focus cues are given. Therefore, an investigation on the effects of attentional focus strategies on the learning of a novel task, while comparing groups that receive reminders of the intended focus strategy at different frequencies, is warranted. Based on the previous literature (see Table 1), the three most commonly used reminder frequencies are every two, four or ten trials. 


\section{Statement of the Problem}

While previous studies on the differences of external and internal focus strategies have resulted in a general consensus that an external focus leads to better learning, there has been no consensus as to how often to remind subjects of their focus strategy. The purpose of the study was to examine the effects of an external focus of attention on the learning of dart throwing at three different focus reminder rates (every two, every four and every ten trials).

\section{Hypothesis}

The hypothesis is that there will be no difference in performance between the three groups during skill acquisition, but that the group given reminders after every fourth trial will perform better than the groups given reminders after every second and tenth trial during both the delayed retention and transfer tests. Similar to the idea of the guidance hypothesis, too many reminders will hinder learning while too few reminders will lead the subjects to lose focus on the intended cue.

\section{Delimitations}

This study was delimited to the following parameters.

1. None of the participants had previous practiced dart throwing on more than five different occasions.

2. Participants were between ages 18-26.

\section{Assumptions}

This study was based on the following assumptions.

1. All of the subjects were motivated to participate to the best of their ability. 
2. All of the subjects were honest about their previous experience with dart throwing.

3. It was assumed that all of the subjects followed the given focus strategy while completing the task.

\section{Limitations}

This study was limited by the following factors.

1. Population was limited to college kinesiology students.

2. The data collector was not blind to the study.

3.

\section{Definition of Terms}

The following terms are defined as used in this study.

Attentional Focus Strategy. The directing of attention to specific characteristics in a performance environment, or to action preparation activities (Magill, 2007).

External Focus. A focus that directs one's attention towards the effects and/or outcome of one's movements (Magill, 2007).

Internal Focus. A focus that directs one's attention towards the body part or parts involved in a movement (Magill, 2007). 


\section{Chapter 2}

Review of Literature

The purpose of this review is to evaluate the findings of past research on the use of attentional focus strategies during the learning of novel skills. An emphasis is placed on recent research explaining the advantages of both an external and an internal type of attentional focus. Additionally, research conducted on differences between novices and experts, differences in the frequency at which focus cue reminders are given, and research using dart throwing as a learning task is discussed.

Wulf and Weigelt (1997) investigated the influence of instructions that elicit an internal focus on the learning of a complex motor skill. Their study included two experiments. The first experiment examined learners' performances when given attentional focus instructions compared to no attentional focus instruction. Eighteen subjects (6 females and 12 males) were randomly assigned into two groups so that there was an equal number of males and females in each group. The skill to be learned was a full-body, slalom-type movement on a ski simulator. The ski simulator is a device that is comprised of a platform with wheels that sits on two bowed rails and is attached by elastic rubber belts. As the participant pushes on the platform in either direction along the horizontal axis, the platform rolls from side to side. As the platform moves to the ends of the rails, the strain on the rubber belts forces the platform to return to the center position. The goal of the task was to move the platform in oscillatory movements along the rails with as large an amplitude and as high a frequency as possible. 
Subjects had no previous experience with the task and practiced the task for three consecutive days, which resulted in 20 total trials: seven 90 second trials on Day 1, six trials on Day 2 and seven trials on Day 3 (the first trial on Day 1 was considered a pretest). There was a 90 second rest period between each trial. The reported performance measurement was the product of the movement amplitude (total distance the platform moved in centimeters) and frequency (the number of complete cycles the platform moved from side to side per second, reported in hertz) during each trial. A metronome set the target frequency at $0.5 \mathrm{~Hz}$.

The two experiment groups consisted of an attentional focus group and a control group. Participants in the attentional focus group received instructions to focus on regarding the optimal timing to apply force to the platform. The instructions were to exert force on the platform after it had passed the center of the ski simulator. This type of instruction elicited an internal focus of attention. Participants in the control group did not receive any instructions and were allowed to discover the correct movement on their own. Before the last trial, a situation of stress was induced by informing all subjects that they were to be evaluated by a skiing expert. Scores from trials 1, 2, 7, 8, 13, 14, 19 and 20 were analyzed in a 2 (group) x 3 (Day) x 2 (Trial) ANOVA. The pretest and "stress" test scores (trials 1 and 20) were analyzed separately.

Results demonstrated that both groups performed similarly at the beginning of practice (no significant difference between groups on the pretest) and that both groups improved over the course of practice (the main effect of both day and trial were significant). While, the control group demonstrated greater improvement than the attentional (internal) focus group during practice, the group and trial interaction only 
approached significance. However, the control did perform significantly better than the attentional (internal) focus group on the "stress" test. The researchers concluded that the use of instructions (internal focus) compared to no instruction proved to be detrimental to learning of the given task. Furthermore, the advantage of no instruction was more pronounced under stressful conditions.

In the second experiment of this study, the researchers examined the effects of giving instructions on the optimal timing of force application later in practice. Nine female university students (whom did not participate in the first experiment) were randomly selected to participate in this portion of the study. The task and the apparatus were exactly the same as in the first experiment. All of the subjects practiced the task for three consecutive days (seven trials on Day 1, six trials on Day 2, and six trials on Day 3) and then returned one week later to complete a three trial retention test. After the retention test, all of the participants were given similar instructions (internal attentional focus) as those given in the first experiment on the timing of force application to the platform and three more trials were performed, totaling six trials on Day 4. The data were analyzed using a 4 (day) x 2 (first vs. last trial per day) ANOVA.

The participants showed improvement both over the course of practice and throughout each day. Both the main effects and the interaction of day and first trial versus last trial were significant. Although the subjects demonstrated improvement from end of Day 3 to the beginning of Day 4, performance dropped once an internal focus was given. The scores on the last three trials of Day 4 (with an internal focus) were worse the last three trials on Day 3 (no internal focus). Wulf and Weigelt concluded that instructions (internal focus) led to decreased performance when introduced later in 
learning as well. Furthermore, the researcher's concluded that learning by discovery might be more effective than learning with instructions that direct the learner's attention to a particular aspect of the movement and that an internal focus may be detrimental to learning.

Wulf, Prinz and Hob (1998) further examined the effects of using different attentional focus strategies in the learning of a novel task. The main purpose of their study was to investigate whether it is more effective to give instructions that direct attention toward a learner's body movements (internal focus) or to give instructions that direct attention to the effects of the learner's body movements (external focus). The researchers divided the study into two experiments. In the first experiment, they used the same ski simulator as the one Wulf and Weigelt (1997) used for experimentation. They randomly assigned thirty-three people (13 males and 20 females) to one of three groups: an internal-focus, an external-focus and a control group. The internal-focus group was instructed to exert force on their outer foot as they moved back and forth on the simulator. The external-focus group was instructed to exert force on the outer wheels of the simulator. The control group was not given any specific instructions. Testing was conducted on three consecutive days with 8 trials on each of the first two days of testing. Throughout testing, instructions were repeated every other trial. Six retention trials were conducted without instruction on Day 3. Much like the Wulf and Weigelt (1997) study, the amplitude and frequency were analyzed for the first and last trial of each day (including the retention test). The practice data were analyzed using a 3 (group) x 2 (day) x 2 (trial) ANOVA and the retention data were analyzed using a 3 (group) x 2 (trial) ANOVA. 
During practice, all groups showed increases in movement amplitude (the main effets of both day and trial were significant). In addition, the interaction between day and trial was significant, indicating that improvements were greater on Day 1 than on Day 2. However, the external-focus group showed the greatest amplitude increase while the internal-focus group showed the smallest increase. Post-hoc tests indicated that the external-focus group was significantly more effective than the internal-focus group and that the internal-focus group was significantly less effective than the other two groups. Furthermore, movement frequency severely decreased and plateaued from the first trial to the last trial (the external-focus ended up with the lowest frequency and the control group had the greatest decrease in frequency). While the main effects and interactions of day and trial were significant, the main effect of group and the interactions involving group did not show significance. During the retention tests, the external-focus group scored significantly higher amplitudes than the other two groups and all groups demonstrated significant improvement. Both the control and external-focus groups showed increases in frequencies, however, the improvements were not significant. The main effect of group and the group and trial interaction failed to reach significance.

The researchers concluded that the external-focus group was significantly more effective than the internal-focus group. Furthermore, the use of an internal-focus resulted in a degradation of performance when compared to the external-focus group and the control group. However, the degrading effects of an internal focus were not permanent, as evident by the similar results between the internal-focus group and the control group during the retention tests. 
In experiment two, the researchers examined the generalizability of the findings in experiment one, which indicated that an external focus results in greater performance when compared to an internal focus or no instructed focus at all, by replicating the experiment with a new novel task. Sixteen university students were randomly selected to participate in this part of the study. In this experiment, the apparatus was a stabilometer (which consists of a wooden platform that swings about an axis) and the novel task was to balance oneself on the stabilometer by keeping the instable wooden platform as horizontal as possible. The root mean squared (RMS), measured by the degrees from the horizontal, was the dependent measure. In this experiment there was no control group. The internal-focus group was instructed to try to keep their feet level and at the same height, while the external-focus group was instructed to keep red markers, which were placed on the platform near the feet, level and at the same height. Seven practice trials were conducted on each of two consecutive days of testing with 90-second breaks between trials. Again, participants were verbally reminded of their focus before every other trial. Retention tests were conducted on the third day of the experiment.

The RMS error decreased for both groups over the two days of practice, however, the differences between the two groups were very minimal (the external-focus group tended to have larger errors on Day 1). The main effects for day and trial as well as the interaction for day and trial reached significance, however, the main effect for group did not. During the retention tests, when no focus instructions were given, the external-focus group performed significantly better than the internal-focus group. From these findings, the authors were able to conclude that an external focus results in better learning. 
Overall, the authors were able to show the learning benefit of an external focus over an internal focus and the generalizability of said benefits to multiple full-body, novel tasks.

Wulf, Lauterbach and Toole (1999) teamed up to test the aforementioned findings that an external attentional focus is more beneficial in a more practical, real-life training situation. Therefore, the novel task to be learned in this study was a golf pitch shot. Twenty-two right-handed university students (9 women and 13 men) were randomly assigned to one of two groups: internal focus and external focus. Participants were to hit golf balls from 15 meters away onto a 45-centimeter target, surrounded by four concentric circles. The first zone (the center target) was worth 5 points, the second zone was worth four points and so forth. All shots were performed outdoors on a lawn surface using a 9-iron golf club.

Before holding the golf club, participants in the internal focus group were instructed to hold their hands together as if they were holding onto the golf club and to focus their attention to the movement of their arms as they swung them back and forth. They were then instructed to swing the arms back and forth (this time with a golf club) so that the left arm was straight and the right arm was bent during the backswing, both arms were straight during the forward swing and that the left arm was bent and the right arm was straight during the follow through. The participants in the external focus group were instructed to hold the club in the correct grip position and to let the club perform a pendulum-like motion during the swing. To accomplish this, they were instructed to push the club to start a pendulum motion and to let the club swing freely and to focus on the weight, the straight-line direction and the acceleration of the club-head as it moved forward toward the ball. The participants in the external focus group never practiced the 
swing without the golf club in hand. After 10 minutes of instructions and 20 practice shots with the golf club (without hitting a ball), each participant performed 80 trials divided into eight trial blocks. One day later, all participants performed a retention test of 30 shots divided into 3 trial blocks. A 2 (Group) x 8 (Trial Block) ANOVA was used to analyze the practice data and a 2 (group) x 3 (Trial Block) ANOVA was used to analyze the retention test data.

Although both groups increased their accuracy throughout the test, the external focus group performed significantly better during both practice and the retention test (the main effects for both group and block reached significance). Also, the researchers were able to state that the external group had achieved higher scores more frequently and had fewer shots that missed the target completely. Retention test results indicate that the external focus group demonstrated significantly better learning (the main effect of group was significant).

In conclusion, the researchers were able to reaffirm the previous findings that an external focus of attention leads to better learning. Additionally, based on the fact that the external focus group performed better early in practice in this experiment, the researchers suggest that there might be task-related differences affecting the effectiveness of external versus internal focus. Furthermore, compared to the distance between cues in the previous literature, the distance between the internal focus cue and the external focus cue in the present study was greater. Therefore, the researchers also suggest that the distance between the internal and external cues could account for the differences in performance observed earlier in practice. 
Wulf, McNevin, Fuchs, Ritter and Toole (2000) further examined the effects of attentional focus on complex motor skills. In part one of their experiment, the main objective was to examine the differences of using external cues that direct attention to the effects of one's movements or cues that direct attention away from one's movement. Thirty-six individuals (21 women and 15 men) volunteered for the study that required its participants to hit tennis balls at a stationary target. The tennis balls were shot out of a ball machine located on the opposite side of the tennis court and sent at a speed of 30 $\mathrm{km} / \mathrm{h}$ so that the ball bounced 1 meter before the service line. The participants were to hit the ball onto a target that was located on the opposite end of the court. The target had four zones, however, only the center zone was visible to the participant from the opposite end of the court. Participants got four points if their shot reached the center zone, three points for the next zone, two points for the next zone and one point for the last zone. No points were awarded if the ball failed to hit the target. Before testing, the experimenter spent several minutes with each participant describing and demonstrating the basic technique of the forehand serve. Participants were instructed to hit the ball in front of their hip and to follow through the ball. They were allowed to take 10 practice hits using the ball machine.

The authors called one of the groups the antecedent group and the other the effect group. The antecedent group was instructed to focus on the trajectory of the ball coming out of the ball machine coming towards them and to focus on the ball as it made contact with the racket. The effect group was instructed to focus on the anticipated trajectory of the ball as it left the racket and to imagine the ball hitting the target. All participants performed 10 blocks of 10 trials with roughly 1-minute breaks between blocks. 
Reminders of the focus cue were given before the first trial of every 10-trial block. One day after the practice trials, all participants returned for a retention test, consisting of three 10-trial blocks. The total scores from each of the trial blocks were analyzed using a 2 (group) $x 10$ (block) ANOVA for the practice trials and a 2 x 3 ANOVA for the retention test. Although there was no significant difference between the two groups during the practice trials, the effect group scored significantly better during the retention test, indicating higher levels of learning. The researchers concluded an external attentional focus, directed towards the effects of the performers actions, is more beneficial than a focus directed to some other cue when learning a motor skill.

The purpose of part two of the experiment was to examine the effect of an attentional focus on the anticipated effect of one's movements (similar to the effect group of the first experiment) when compared to attentional focus on the immediate effect of one's movement related to the movement pattern or technique of the movement. Twentysix right-handed adults (14 women and 12 men) volunteered for the experiment. The task was again the golf task used by Wolf et al. (1999). The club group was instructed to focus on the swing of the club and to concentrate on letting the club follow a pendulumlike motion. The target group was instructed to focus on the anticipated arc of the ball's trajectory and to imagine the ball landing on the target.

All participants performed 80 trials during the practice trials and returned one day later to perform a retention test consisting of three 10-trial blocks. The total score on each block were analyzed using a 2 (group) x 8 (block) ANOVA for the practice trials and a 2 (group) $\mathrm{x} 3$ (block) ANOVA for the retention test. Although both groups improved during practice, the club group scored significantly higher than the target 
group. Furthermore, the club group also scored significantly higher during the acquisition phase of the study when compared to the target group during the retention test. Conclusions than an external focus directed towards the technique of the movement results in better performance and overall learning of a motor skill.

Due to the conflicting findings on the rates of skill improvement when using an external focus in previous research, McNevin, Shea and Wolf (2003) decided to further investigate the topic using different external foci. Based on a review of the literature, the researchers hypothesized that the further away the external focus of attention was from any body part involved in the movement, the sooner the learning benefits of an external focus over an internal focus of attention were observed. For example, in an attentional focus study examining the learning of a golf swing by Wulf, Lauterback and Toole (1999), benefits of an external focus over an internal focus were observed early in the learning process. In contrast, in the study by Wulf, Prinz and Hob (1998), benefits of an external focus over an internal focus when learning a balancing task were not observed until the retention test. Based on these observations, the present experiment compared different distances between the body and the external focus.

The apparatus and task used in this study were the same as those used in the study by Wulf et al. (1998). Forty university students ( 28 females and 12 males) were randomly assigned to one of four experimental groups: one internal focus group and three external focus groups. Those in the internal focus group were instructed to focus on the their feet. Of the three external groups, those in the "near" group were instructed to focus on markers placed on the platform directly in front of the participant's feet, those in the "far-inside" group were instructed to focus on markers placed between the participant's 
feet and those in the "far-outside" group were instructed to focus on markers placed on either side of the participant's feet.

The objective of the task was to keep the platform as horizontal as possible by focusing on keeping the object of focus as horizontal as possible. However, all participants were instructed to look straight ahead and not down at the markers while performing the task. All participants performed seven 90 -second trials on each of two days, with short reminders of the respective focus given before every other trial. A seven-trial retention test was conducted on the third day of testing. Performance during the practice trials was measured by calculating the root-mean-square error (RMSE) in degrees from the horizontal using a potentiometer. The RMSE score represents the absolute error in performance. For the retention trials, mean power frequency (MPF) was also measured from the waveform created by the movement. The MPF indicates how often and by how much a participant makes corrections; more corrections result in higher MPF scores, which indicate better performance. The RMSE data from the practice trials were analyzed using a 4 (focus) x 2 (day) x 7 (trial) ANOVA. The RMSE and MPF data from the retention trials were analyzed using a 4 x 7 ANOVA.

Findings for this study demonstrate that decreases in RMSE scores (improvement) were observed for all groups across the two-day practice trials. Although the far-inside group had smaller RMSE than the other groups during the practice trials, the main effect of group was not significant. Conversely, both the main effects of day and trial and the interaction of day $\mathrm{x}$ trial were significant. For the retention test, main effects of trial and group were significant. Based on a Dunnett's follow-up comparison test, the researchers were able to conclude that the internal focus group had significantly higher RMSE scores 
than the three external focus groups. In other words, the internal focus group was less accurate than the other groups. Furthermore, the near group had significantly higher RMSE scores than the far-inside and the far-outside groups. Analysis of the MPF of the retention data resulted in a main effect of group. Again, Dunnett's follow up tests showed that the differences between the internal group and the near external group were not significant. However, the far-inside and the far-outside external groups had significantly higher MPF than the other two groups, indicating better performance.

Although the data did not indicate immediate performance benefits of using an external focus that is further from a movement action, enhanced learning using an external focus was observed eventually. From these results, the researchers proposed the "constrained action hypothesis". This hypothesis explains that the lower performance of the internal and near-external focus groups was due to the participants actively attempting to control their posture, which results in the degradation of a natural, dynamic movement. Furthermore, the higher number of small corrections (as seen in the high MPF scores) for the far-inside and far-outside external focus groups compared to the internal focus group suggests that the far-external focus groups might rely more on automatic control processes which result in enhanced learning.

Wulf, McNevin and Shea (2001) further examined the "constrained action hypothesis" in their study. The main purpose of their study was to investigate whether or not an external attentional focus reduces attentional demands and promotes more automatic control processes than an internal attentional focus. They set out to test this by having participants learn a primary novel task while responding to a secondary stimulus. They hypothesized that if the constrained action hypothesis is true, then the external 
focus group should perform better on the primary task while scoring better on the secondary task. For their study, 28 university students (23 females and 5 males) were randomly assigned to either an internal or external attentional focus group. The primary task along with the apparatus was the same balancing task and stabilometer used in the study by Wulf et al. (1998) and McNevin et al. (2003). Much like in the McNevin et al. (2003) study, task performance was measured using RMSE in degrees and MPF from the waveforms created by the movement. The secondary task required participants to stop an auditory stimulus by pressing down on a hand held plunger. The stimulus was presented eight times during each trial with at least five seconds but no more than 16.75 seconds between stimuli. Reaction time (RT) was recorded as a way to score performance on the secondary task.

Similar to the study by McNevin et al. (2003), the internal focus group was instructed to focus on keeping their feet balanced and horizontal and the external focus group was instructed to focus on keeping markers (placed 22 centimeters away from participants' feet) balanced and horizontal. All participants were instructed not to look at their feet or the markers during the trials. Participants were informed that the primary task was to stay balanced on the stabilometer and that the reaction to the auditory stimulus was only a secondary task. To make sure that the stabilometer task was not influenced by the concurrent performance of the secondary task, each participant performed one trial of the primary task without the secondary task on each of the three days of testing, so that six of the seven trials on each day included both tasks. Testing consisted of two days of seven practice trials followed by a third day of seven more retention trials. Before each practice trial, participants were verbally reminded where to 
focus their attention; there were no reminders during the retention trials. Also, each participant performed one set of baseline reaction time trials (without the stabilometer task) at the beginning and end of each day of testing.

Practice data were analyzed with a 2 (focus) x 2 (day) x 6 (trial) ANOVA. Also, a 2 (focus) x 2 (task with vs. without secondary task) x 2 (day) ANOVA was conducted to determine if the secondary task influenced the performance of the primary task during the practice trials. While both groups showed improved RMSE scores over the two days of practice (significant main effect for day, trial and interaction for day and trial), there was no significant difference between the two groups. Also, there was no significant difference between found between completing the primary task with and without the secondary task. While RT scores for the secondary task were the same for both groups at the beginning of the study, RT scores did show significant decreases over practice (significant main effects were observed for group, trial and day). The retention data were analyzed using one-way ANOVAs for RMSE, RT and MPF data. Also, average RMSE on trials with the secondary task were compared to those without the secondary task using additional one-way ANOVAs. The external focus group showed significantly smaller RMSE scores. The analysis run on RMSE of trials with and without the secondary task did not show any significant differences. MPF scores were significantly greater for the external group. Finally, RT scores for the external group were significantly lower during the retention test. Based on the RMSE and RT scores, the authors were able to conclude that not only did an external focus promote better learning of the primary task, but it also put less attentional demand on the participant. The lower 
RT scores for the external focus group provides further evidence support the constrainedaction hypothesis.

Perkins-Ceccato, Passmore, and Lee (2003) investigated the different effects of using an external versus and internal focus with subjects of different skill level. Using a golf pitch-shot as the novel task, the researchers predicted that the detrimental effects of an internal focus of attention would be more detrimental for high-skilled golfers and that low-skilled golfers would perform better using an internal focus of attention. Ten highskilled male golfers and 10 low-skilled golfers (eight male and two female) were randomly selected to participate in the study. The high-skilled golfers had a mean handicap of four, while the low-skilled golfers had a handicap of 26. Furthermore, none of the low-skilled golfers had ever received formal golf training all participants had played recreationally for at least two years. The object of the task was to pitch a standard sized golf ball, using a 9-iron golf club, towards a fluorescent orange pylon target from four different distances $(10,15,20$ and 25 meters). four target areas were created using yellow nylon rope. Four target areas were created using yellow nylon rope and the golf balls were coated with white chalk, so that the distance and angle of the landing spot from the target could be measured in order to calculate two-dimensional error scores using $\mathrm{x}$ - and y-coordinates. All pitch-shots were taken on a flat grass field.

All participants used both external and internal attentional focus strategies during the trials. Forty shots were taken using one focus strategy, followed by another 40 shots taken using the other focus strategy, for a total of 80 pitch-shots. The order of focus strategy used and distance shot from were both counterbalanced and in a quasi-random order. All subjects were allowed to take 5 warm-up shots at different locations, other 
than the ones used for testing, before the trials began. Also, all subjects wore vision occlusion goggles that were manually controlled to turn opaque once the subject swung and made contact with the ball. After each shot, participants were asked to estimate their performance based on the attentional focus strategy they were using. The purpose of this was to encourage the participants to adopt the given focus strategy and no score was recorded of their estimation. Two-dimensional average error scores and variable error scores (which calculated the variability of each participants error with the mean error of their group) were recorded and calculated using a 2 (skill) x 2 (attentional focus) x 2 (attentional focus order) x 4 (distance) ANOVA. Furthermore, a 2 (skill) x 2 (attentional focus) $\mathrm{x} 4$ (distance) ANOVA was used to analyze only the data from the first attentional focus condition (the first 40 trials).

The researchers only found main effects for skill and distance from the results of both ANOVA tests for average error score. The high-skill group had significantly lower average error scores than those of the low-skill group. Also, average error score significantly decreased with decreases in target distance. The researchers attributed the lack of significant interactions in the analysis to the possibility that once a participant was given a focus strategy, the effects of the initial strategy influenced the participants' performance during the second set of 40 trials. The ANOVA results that analyzed the variable error scores also revealed significant main effects for skill and distance similar to those found for the average error scores. For the first ANOVA, there was also a significant interaction between skill and attentional focus order. The low-skill group produced lower variable error when completing the task with an internal focus first; the high-skill group produced lower variable error when completing the task with an external 
focus first. From the second ANOVA, the researchers were able to deduce that the highskilled group produced lower variable error scores when using an external focus than did the low-skilled group. Also, the two skill groups were not significantly different under the internal focus condition.

The researchers concluded that the skill of an individual has an affect on the effectiveness of attentional focus strategies used in a golf pitch shot. This conclusion was supported by their results, which demonstrated that low-skilled golfers not only performed more consistently when using an internal focus before using an external focus, but also that low-skilled golfers performed better when using just an internal focus as compared to just an external focus. Furthermore, the performance of high-skilled golfers was significantly better when an external focus strategy was used as compared to an internal focus strategy. The researchers acknowledged that these findings could be due to the wording of the attentional focus strategies and that further investigation is warranted.

Zachry, Wulf, Mercer and Bezodis (2005) investigated the effects of an external or internal focus on a basketball free throw shot. Specifically, the investigators examined both shooting success and electromyography (EMG) readings when assessing overall performance. Unlike previous studies examining the effects of attentional focus strategies via EMG activity (Vance, Wulf, Tollner, McNevin \& Mercer, 2004), this study used a sport activity with a clear goal and outcome.

In the study, 14 university students (6 females and 8 males) with at least 1 year of high school team or physical education class basketball experience participated. All participants (regardless of experience) were given instructions on correct free throw technique and were given unlimited practice before data collection was to begin. To 
record the EMG activity, electrodes were placed over the medial biceps brachii, the long head of the medial triceps brachii, the medial deltoid and the medial flexor carpi radialis of each participant's shooting arm. Participants were instructed to get in their position, "get set" and then to "go". The first movement after the word "go" was considered onset of movement and used as the starting point for movement analysis. The shooting performance was assessed by a scoring system that awarded five points if the ball successfully went through the hoop, three points if the ball touched the hoop, two points if the ball touched the backboard and hoop and one point if the ball touched only the backboard. No points were awarded if the ball missed the hoop and backboard completely. All shots were taken with a deflated women's size basketball (to protect the laboratory equipment).

All participants shot free throws under both external and internal focus conditions. For the internal focus condition, subjects were told to focus on snapping their wrist during the follow through of the free throw shot. For the external focus condition, subjects were told to focus on the center of the rear of the basketball hoop. All subjects were given reminders after every trial for the first three trials and then after every two trials thereafter. Participants completed two sets of 10 trials for each condition with at least one minute of rest between each set and at least 30 seconds of rest between each shot. The attentional focus order was counter-balanced between participants and all participants were filmed via camcorder positioned perpendicular to the participant' sagital plane. The raw EMG data was used to calculate root-mean-square (RMS) values from the onset of movement to three video frames after ball release. The RMS EMG data from the first and last trial of each block was analyzed in a 2 (attentional focus) $\mathrm{x} 2$ 
(block) x 2 (trial) ANOVA, with repeated measures on all factors. Performance accuracy was analyzed in a paired t-test.

From the results of the analyses, the researchers found significant findings in both performance accuracy and EMG activity. Participants scored significantly better when shooting with an external focus than when shooting with an internal focus. Also, while no significant differences were found between EMG activity for the flexor carpi radialis or the deltoid muscles under the two conditions, participants produced significantly less activity in the triceps and biceps brachii muscles during the external focus condition. No other interactions or main effects reached significance. The authors concluded that this study further confirms and extends the findings of previous research on attentional focus strategies that an external focus of attention leads to better performance outcomes, as well as movement economy.

Marchant, Clough and Crawshaw (2007) tested the effects of attentional focus strategies using dart throwing as the novel task. The aim of the study was to replicate the findings of Radlo et al. (2002) that an external focus is more beneficial to learning dart throwing than an internal focus, while also measuring participants' perceived difficulty with the focus strategies. The researchers believed that the focus instructions used by Radlo et al. (2002) were more complicated than those used in studies by Wulf and colleagues. Marchant et al. (2007) hypothesized that perceived difficulty of a focus strategy may have an effect on performance. As recommended by Radlo et al. (2002), the current study also included a control so that a full comparison between focus strategies.

The task and apparatus (as well as much of the procedures and inclusion criteria) were the same as used by Radlo et al. (2002) and consisted of a target printed on a white 
paper target with ten concentric rings. The center ring was 10 centimeters in diameter and each successive ring was 10 centimeter greater in diameter. The center target was worth zero points and the outer most circle was worth nine points. The target was placed 366 centimeters from the thrower and 183 centimeter from the floor.

A total of 67 university students and staff (37 females and 30 males) with an age range of 18 to 51 and mean age of 20 years participated in the study. In order to be included in the study, participants were to have not thrown darts on more than five previous occasions and score less than an average of $3.5 / 9$ on five practice throws. Participants were randomly assigned to one of the three focus groups: control, internal and external. The focus instructions given to the internal group were to "feel the weight of the dart in their hand, think about drawing the dart back to the ear, feel the bend in the elbow and feel the dart as it left the finger tips". The focus instructions given to the internal group were to "focus on the center of the dart board, slowly begin to expand upon perspectives of the dart board, then refocus to the center of the dart board, expanding the center and making it as large as possible and toss the dart when so focused". The control group received no focus strategy and was told to perform as best they could. Participants received no instructions on technique and they were reminded of their intended focus after every two trials.

Participants were allowed to take 10 practice throws using the given focus strategy, before performing 40 test trials (divided into 10 trial blocks), followed by a post-task questionnaire. The questionnaire consisted of five questions and was based on a 5-point Likert. The questions were, "How difficult was it to the carry out these instructions, How difficult was it to maintain these instructions throughout the task, What 
was the level of the mental demands, How successful do you think these instructions were in making your dart throws accurate and, Approximately, on how many of the throws do you think you used the instructions?" The last question was answered based on a 0 to 100 percentage score. The mean score from the trial blocks was used to measure accuracy in a 3 (attentional strategy) x 10 (trial block score) ANOVA. Each question in the post-task questionnaire was analyzed using separate one-way ANOVAs.

\section{Summary}

An overwhelming amount of the previous literature on attentional focus strategies conclude that an external focus of attention results in better learning when practicing a novel skill. Furthermore, all of the studies on attentional focus strategies give participants reminders of the intended focus (Table 1 provides a brief summary of attentional focus studies, the number of trials tested and the reminder frequencies used). However, no study has given a reason for the rate the reminders are given, nor has there been a generally accepted rate that leads to the highest performance and learning outcomes. Also, the majority of the studies have given participants a significant amount of practice before trials were recorded. Therefore, the purpose of this study is to to examine the effects of an external focus of attention on the learning of dart throwing at three different focus reminder rates (every two, every four and every ten trials). It is hypothesized that, while there will be no difference in performance between the three groups during skill acquisition, the group given reminders after every fourth trial will perform better than the groups given reminders after every second and tenth trial during both the delayed retention and transfer tests. 
Table 1

Summary of attentional focus studies.

\begin{tabular}{|c|c|c|c|c|}
\hline Study & Task & $\begin{array}{l}\text { Trials } \\
\text { (Days) }\end{array}$ & $\begin{array}{l}\text { Reminder } \\
\text { Freq }\end{array}$ & Conclusion $^{\mathrm{a}}$ \\
\hline \multirow[t]{2}{*}{ Wulf et al., 1997} & i) Ski simulator & $20(3)$ & 4 & Non- $\mathrm{I}^{\mathrm{b}}$ \\
\hline & ii) Ski simulator & $19(3)$ & N/A & Non- $\mathrm{I}^{\mathrm{b}}$ \\
\hline \multirow[t]{2}{*}{ Wulf et al., 1998} & i) Ski simulator & $16(2)$ & 2 & $\mathrm{E}>\mathrm{I}$ \\
\hline & ii) Stabilometer & $14(2)$ & 2 & $\mathrm{E}>\mathrm{I}$ \\
\hline Shea et al., 1999 & Stabilometer & $14(2)$ & 2 & $\mathrm{E}>\mathrm{I}$ \\
\hline Wulf et al., 1999 & Golf chip shot & $80(1)$ & 10 & $\mathrm{E}>\mathrm{I}$ \\
\hline Wulf et al., 2001 & Stabilometer & $14(2)$ & 1 & $\mathrm{E}>\mathrm{I}$ \\
\hline Radlo et al., 2002 & Dart throwing & $40(1)$ & 1 & $\mathrm{E}>\mathrm{I}$ \\
\hline \multirow[t]{2}{*}{ Wulf et al., 2002} & i) Volleyball serve & $50(2)$ & 5 & $\mathrm{E}>\mathrm{I}$ \\
\hline & ii) Soccer loft kick & $30(1)$ & $1 \& 3$ & $\mathrm{E}>\mathrm{I}(1>3)$ \\
\hline McNevin et al., 2003 & Stabilometer & $14(2)$ & 2 & $\mathrm{E}>\mathrm{I}$ \\
\hline Zachry et al., 2005 & Basketball shot & $40(1)$ & 2 & $\mathrm{E}>\mathrm{I}$ \\
\hline \multirow[t]{2}{*}{ Poolton et al., 2006} & i) Golf putting & $30(1)$ & 3 & No diff \\
\hline & ii) Golf putting & $30(1)$ & 3 & No diff \\
\hline Marchant et al., 2007 & Dart throwing & $40(1)$ & 2 & $\mathrm{E}>\mathrm{I}$ \\
\hline \multirow[t]{2}{*}{ Emanuel, et al., 2008} & Dart throwing & $50(1)$ & 10 & E > I (Adults) \\
\hline & & & & $\mathrm{E}<\mathrm{I}$ (Children) \\
\hline Marchant et al., 2009 & Dart throwing & $40(1)$ & 4 & $\mathrm{E}>\mathrm{I}$ \\
\hline Bell et al., 2009 & Golf chip shot & $50(1)$ & 10 & $\mathrm{E}>\mathrm{I}$ \\
\hline
\end{tabular}

\footnotetext{
${ }^{\mathrm{a}}$ External Focus (E) or Internal Focus (I)

${ }^{b}$ The non-internal group
} 


\section{Chapter 3}

Methods and Procedures

\section{Restatement of the Purpose}

The purpose of this study was to examine the effects of differing external focus cue reminder rates on the learning of dart throwing in novices.

\section{Subjects}

Twenty-four students (20 female and 4 male) at the California Polytechnic State University at San Luis Obispo, ranging in age from 18 to 26 years, volunteered to participate in this study. Similar to the study by Radlo et al. (2002), all participants were classified as novices based on self-reports that none had thrown darts on more than five previous occasions nor had received any instructions on dart-throwing technique. Informed consent was obtained before any instruction or testing was performed. Participants were randomly assigned to one of three focus cue reminder groups.

\section{Apparatus and Task}

The apparatus consisted of a target printed out on white paper and placed on a corkboard. The corkboard was securely mounted on a wall. The target was 50 centimeters in diameter and had five concentric rings, all of equal area, creating five scoring zones. The center ring was 10 centimeters in diameter, the next outer ring was 20 centimeters in diameter, the next outer ring was 30 centimeters, the next outer ring was 40 centimeters in diameter and the most outer ring was 50 centimeters in diameter. Each scoring zone was given a point value, with the center zone having the highest point value of five points and the most outer zone having the lowest point value of one point. 
Precautions were taken to minimize damage to the un-corked portion of the wall from arrant throws. A diagram of the wall, the corkboard and the dartboard with the scoring zones can be viewed in Figure 1 (not to scale). The dartboard was positioned so that the center bull's-eye was 1.73 meters from the ground. The line from which the participants were to throw the darts was designated with a piece of blue tape placed a distance of 2.37 meters from the front face of the dartboard to the back of the back edge of the blue tape. The darts that were used were of standard regulation length (30.5 centimeters) and weight (50 grams). All measurements and distances of the dartboard and testing area as well as the size and weight of the darts were taken from World Darts Federation rulebook.

Figure 1

Wall with Corkboard and Dart Board

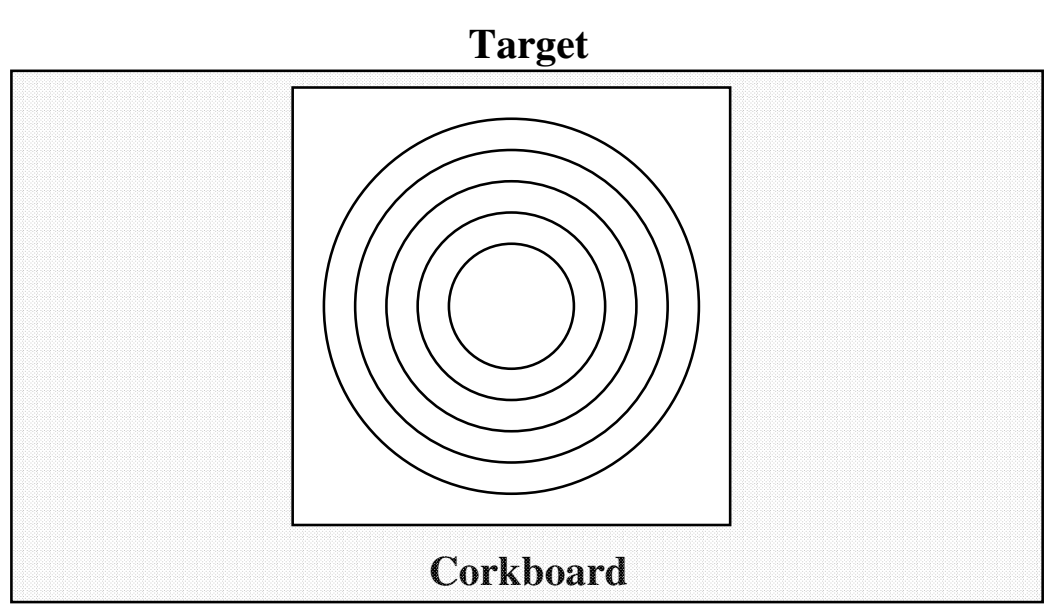

Wall 


\section{Procedures}

Upon arrival at the testing facility, participants completed the informed consent. Participants were then allowed to become comfortable with the laboratory, its surroundings and the position of the dartboard. Opportunity was given for participants to ask questions before instructions were given and testing began. Subjects were randomly assigned to one of three attentional focus practice groups. The difference between the groups was the rate at which the participants were reminded of the external focus cue. One group was reminded of the focus cue after every two trials (2-trial reminder group), one group was reminded of the focus after every four trials (4-trial reminder group) and one group was reminded of the focus after every 10 trials (10-trial reminder group).

All of the subjects received brief instructions and demonstrations on how to hold the dart and the proper over-hand arm movement used to throw the dart. Participants were told to hold the dart with between their thumb and index and middle fingers as if holding a pencil. They were then instructed to hold the dart at eye level while creating roughly a 90-degree angle at the elbow. For the throw, participants were instructed to cock the upper arm back, creating roughly a 45-degree angle at the elbow, and then accelerate the arm forward, so that the dart follows a parabolic curve towards the dartboard. Participants were also instructed that the shoulder should not move throughout the throw and the follow through should result in the arm pointing straight at the target.

Participants were given the same external focus cue at the beginning of testing and the same focus reminders throughout the trials. The focus cue instructions were adapted from the four-step instructions given to participants in a study by Radlo, et al. 
(2002). The changes allowed for a shorter reminder, while still directing attention to key body movements in the dart-throwing motion. The external focus cue was to, "Focus on the center target, slowly expand your focus to the entire board, then re-focus on the center target, making it as large as possible". The wording of the external focus cue stayed the same throughout testing. The primary researcher gave the instructions and demonstration as well as the focus reminders and was the only person to interact with the subjects throughout testing.

Skill acquisition trials were conducted on two consecutive days. On each day of acquisition, participants conducted 30 trials. Therefore, there were a total of 60 trials. All participants were allowed to take three practice tosses before the 30 trials began on each day. After every five throws, scores were recorded and darts were removed from the board. The total possible score obtained for each trial block was 25 points. Five days after the second day of acquisition, the participants returned to perform delayed retention and transfer tests, both consisting of five trials each. All participants were instructed to refrain from practicing dart throwing between acquisition and the retention and transfer tests. For the transfer test, the participants threw darts at a target of same size and height as in acquisition and the retention test, but from a distance of 3.37 meters away from the dartboard. Just like during the two days of acquisition, each participant was allowed to take three warm-up tosses before the test session began. Although the practice throw scores were recorded, they were not analyzed to allow for warm-up decrement. No instructions on technique or attentional cues or verbal feedback were given during the retention test or transfer tests. Half of the subjects within each group took the retention test before the transfer test. 


\section{Data Analysis}

Score on the dartboard was the dependent measure used to assess performance and learning. The highest possible score per trial was 5 points, totaling 25 possible points per trial block. The total score from each trial block was averaged and these averages were used in data analysis. Practice trial data were analyzed using a 3 (group) x 2 (day) x 6 (trial block) analysis of variance (ANOVA) test with repeated measures on the last factor. Separate one-way ANOVAs were run on the delayed retention and transfer tests. Tukey's post-hoc comparisons, with an alpha level of 0.05 , were performed when the results from the practice and retention test ANOVAs reached significance. The statistical software Minitab ${ }$ was used for all statistical analyses. 


\section{Chapter 4}

Results

The purpose of this study was to examine the effects of an external focus of attention on the learning of dart throwing at three different focus reminder rates (every two, every four and every ten trials). Based on the idea of the guidance hypothesis (Schmidt, 1970), it was hypothesized that the group that received a reminder after every fourth trial would perform significantly better than the other two groups during the retention and transfer tests, but that there would be no difference between the three groups during acquisition.

The dependent measure was the dartboard score. The dartboard was comprised of five concentric circles with differing point values. The center circle had the highest point value of five points and the outer-most circle had the lowest point value of one point. No points were awarded for darts that missed the dartboard completely. Acquisition data, retention test data and transfer test data were analyzed separately. The 60 total acquisition trials were completed over two consecutive days (30 trials were completed each day). The acquisition data were analyzed using a 3 (reminder group) x 2 (day) x 6 (trial block) ANOVA. The five retention and five transfer trials were averaged into one trial block per test and analyzed using a one-way ANOVA. An alpha level of 0.05 was used for significance for all tests. Tukey's post-hoc comparisons were performed whenever the analyses reached significance. A graph of the acquisition data trial blocks, along with the retention and transfer trial blocks, for all three groups is presented in Figure 3. 


\section{Acquisition}

Analysis of the $3 \times 2 \times 6$ ANOVA found a main effect for group $(\mathrm{F}=13.61$; $\mathrm{p}<0.001)$. The 4-trial (mean=4.179) and 10-trial (mean=4.226) reminder groups performed significantly better than the 2-trial reminder group (mean=3.897). However, there was no significant difference between the 4-trial reminder group and 10-trial reminder group. There were no other main effects nor were there any interactions between the three factors for the acquisition data.

Table 2

Acquisition Trial Block Scores \& Standard Deviation

\begin{tabular}{|c|c|c|}
\hline Group & Mean Score & Stnd Dev \\
\hline Reminder Every 2 & 3.897 & 0.1403 \\
\hline Reminder Every 4 & 4.179 & 0.1520 \\
\hline Reminder Every 10 & 4.226 & 0.0997 \\
\hline
\end{tabular}

In addition to the statistical findings, graphical representation of the data also shows some interesting trends. The statistics presented in table 2 suggest that the 10-trial reminder group also performed more consistently and with less variation than the other two groups. Although not significant, it appears that this group was able to steadily improve over the course of acquisition with less variability in performance accuracy when compared to the other two groups. This trend is also visible when looking at both Figure 2 and Figure 3. Though the 4-trial reminder group and the 10-trial reminder group did not differ significantly during acquisition, the trend lines in Figure 2 show that the 4- 
trial reminder group performed better at first, but then performed progressively worse over the two days of acquisition.

Figure 2

\section{Acquisition Blocks with Trend}
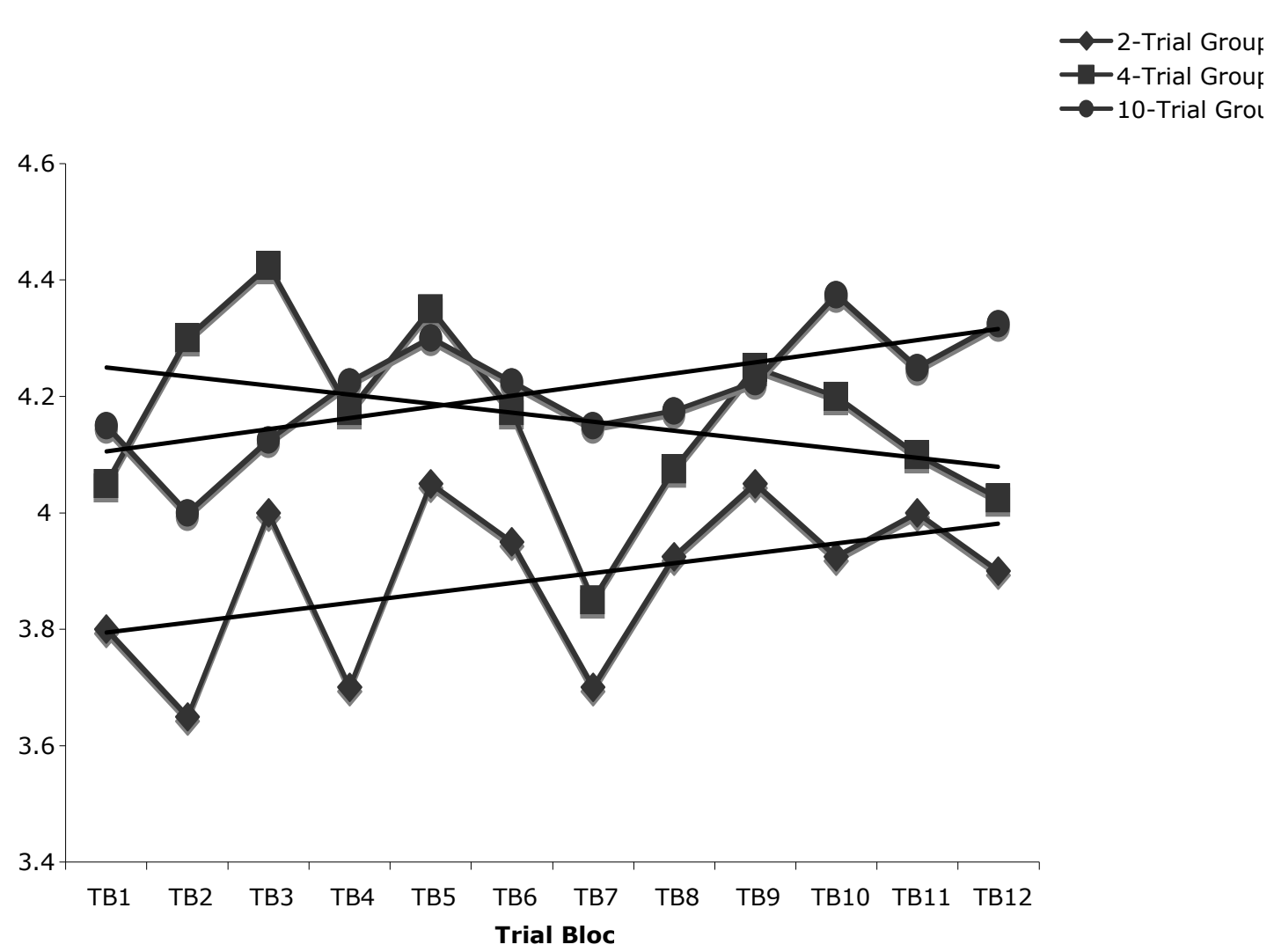

Retention Test

The delayed retention test was performed exactly five days following the acquisition phase. The retention test consisted of five throws at the same distance as acquisition and the subjects were given no focus statement or feedback during the test. Results from the one-way ANOVA showed a significant difference between the three groups $(\mathrm{F}=6.37 ; \mathrm{p}=0.007)$. Post-hoc follow-up tests revealed that the 10 -trial reminder group (mean $=4.6$; std dev=0.338) performed significantly better than the 2-trial reminder 
group (mean=3.925; std dev=0.427). The 2-trial reminder group and the 4-trial reminder group (mean=4.175; std dev=0.377) were not significantly different. The 4-trial reminder group and the 10-trial reminder group also did not perform significantly different from each other. Statistically, however, the difference between 4-trial reminder group and the 10-trial reminder group approached significance.

Figure 3

\section{Acquisition \& Retention and Transfer}

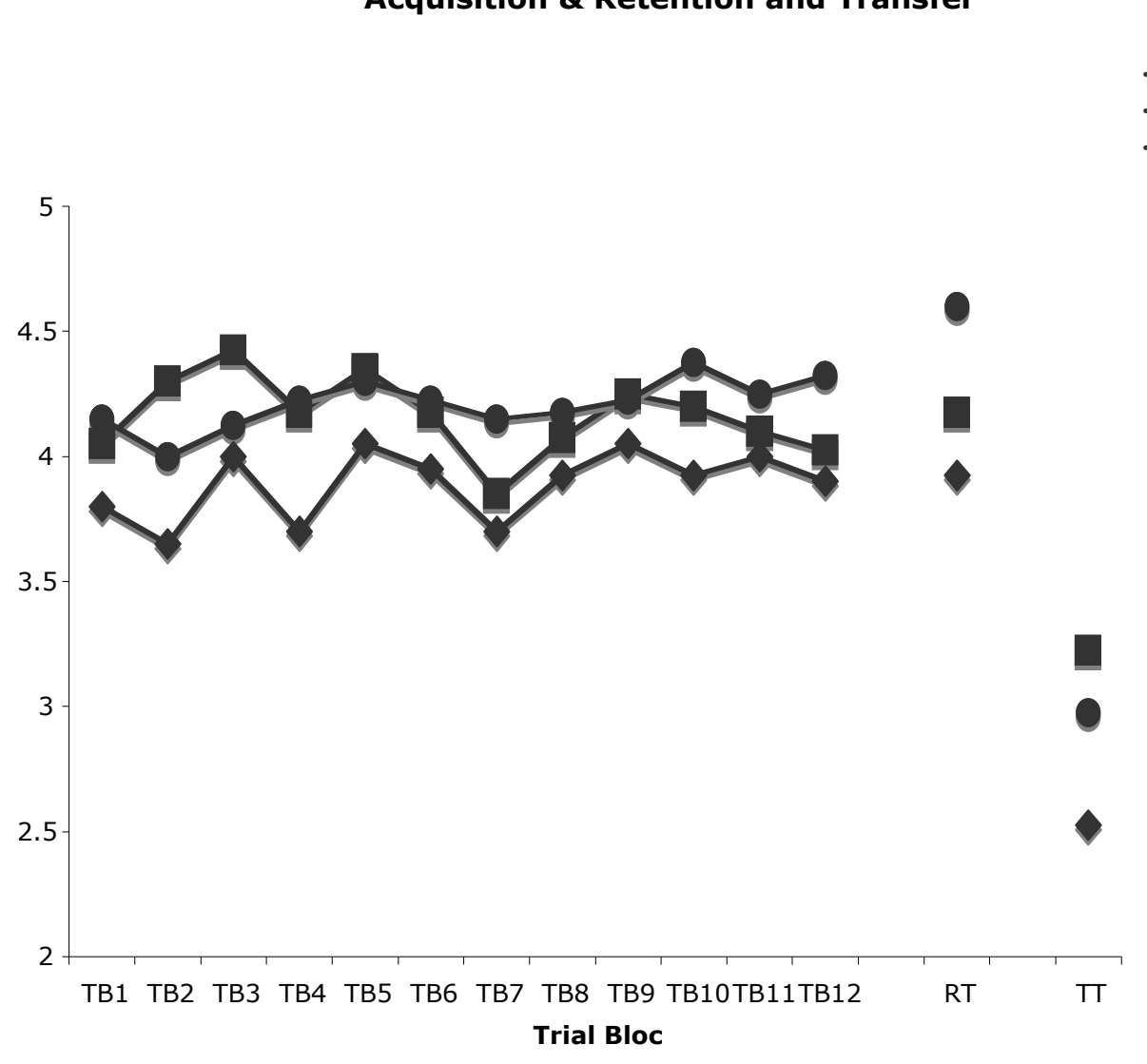

\section{Transfer Test}

The delayed transfer test was also performed five days after the second day of acquisition and required the subjects to throw darts at a target of same size and height as in acquisition, but from a further distance. Again, subjects did not receive any focus 
reminders or feedback during the transfer test trials. The one-way ANOVA failed to find a significant difference between any of the three groups. Similar to the retention test findings, the 4-trial reminder group (mean=3.225) and the 10-trial reminder group $($ mean=2.975) did perform noticeably better than the 2-trial reminder group (mean=2.525). Also, the average group variability during the transfer test $(0.693)$ was almost twice that of the retention test $(0.381)$ and more than five times greater than that during acquisition (0.131).

Table 3

Transfer Test Mean Scores \& Standard Deviation

\begin{tabular}{|c|c|c|}
\hline Group & Mean Score & Stnd Dev \\
\hline Reminder Every 2 & 2.525 & 0.6135 \\
\hline Reminder Every 4 & 3.225 & 0.7126 \\
\hline Reminder Every 10 & 2.975 & 0.7517 \\
\hline
\end{tabular}




\section{Chapter 5}

\section{Discussion}

While previous literature examining the differences between external and internal focus strategies on learning a motor skill have resulted in a general consensus that an external focus leads to better learning (Wulf et al., 1999; Wulf et al., 2000; Radlo et al., 2002; Zachry et al., 2005), studies have varied the number of trials that are performed before reminding the learner of the focus. Furthermore, very little discussion has been given to the frequency of reminder trials. Thus, the purpose of this study was to examine the effects of an external focus of attention on the learning of dart throwing at three different focus reminder rates (every two, every four and every ten trials). The research hypothesis was that the there would be no difference in performance between the three groups during the acquisition phase. However, the 4-trial reminder group was hypothesized to perform better than the other two groups during the delayed retention and transfer tests.

The results of this study showed significant differences in performance accuracy between the groups during acquisition as well as during the retention test, however, not during the transfer test. During acquisition the group that performed the best was the 10trial reminder group. Reminders given after every second and fourth trial seemed to be disruptive and bothersome. Further, the subjects in the groups that received more reminders appeared to be more impatient throughout the acquisition phase. Instead of facilitating the learning process, frequent reminders seemed to interrupt the subjects' concentration. Getting a reminder after every tenth trial, however, seemed to be helpful 
and come at a more useful interval. Although there was no significant main effect for day, there was a visible decrease in performance in the 2-trial and 4-trial reminder groups from the last trial on the first day to the first trial on the second day. Conversely, the 10trial reminder group did not show such a noticeable decrease in performance accuracy.

The results from the retention test provide insight into the effects of differing frequencies of focus reminder trials. The 10-trial reminder group demonstrated the highest levels of learning on the retention test. In fact, the only group that increased accuracy in the retention test was the 10-trial reminder group. The other two groups were more accurate during acquisition than they were during the retention test. And although the difference in accuracy during the retention test between the 4-trial reminder group and the 10-trial reminder group only approached significance, the reason for this could be due to the higher variance in group performance accuracy during the retention test compared to acquisition. Therefore, based on the retention test, the group that was given a reminder after every tenth trial was the most skilled at dart throwing by the end of the study.

The transfer test demonstrated no significant difference between the three groups. The lack of a significant difference here can likely be attributed to the high variance within the groups. The average standard deviation within the three groups for the transfer test block (0.693) was almost twice the average standard deviation for the retention test block (0.381). Also, it is possible that the distance from which the subjects threw darts for the transfer test was too far from the dartboard and the motor pattern for the new throwing distance was not transferable. 
The statistical results coupled with the graphical observations of the data suggest the frequency of reminder trials does affect both performance and learning of a motor skill. The learning process of the two groups that were given reminders more often appeared to suffer compared to the 10-trial reminder group. The high frequency of reminders impeded the subjects from adequately learning the motor pattern necessary to accurately throw darts. The group with the fewest reminders learned the most and was the most skilled at dart throwing at the end of the study. It appears that for dart throwing, more reminder trials are not helpful.

\section{Recommendations}

The findings of this study suggest that there might be an optimal frequency at which subjects should be reminded of their attention focus while learning to throw darts. While it is evident that a focus reminder after every second trial comes too often and that a longer interval between focus reminders is more beneficial to learning, the optimal reminder frequency is still unknown. Furthermore, the results from this study describe a specific population of people. Also, it is unknown whether these findings are replicable with the learning of other, full body motor skills, such as slalom skiing, a golf swing or a basketball free throw. Therefore, further research is warranted to examine both the optimal reminder frequency and the replicability of these findings with other populations of people and with other full body motor skills. Finally, future researchers might want to consider a transfer test distance that is closer to the acquisition and retention distances.

\section{Conclusion}

The group that received a reminder after every tenth trial performed better both during acquisition and the delayed retention test. While this group did not perform 
significantly better than the group that received a reminder after every fourth trial, the data show trends that a reminder every tenth trial could be the most beneficial out of three reminder rates. It is clear, however, that the group that was given a reminder after every second trial performed the worst throughout this study. 


\section{References:}

Bell, J. \& Hardy, J. (2009). Effects of attentional focus on skilled performance in golf. Journal of Applied Sport Psychology, 21(2), 163-177.

Emanuel, M., Jarus, T., \& Bart, O. (2008). Effect of focus of attention and age on motor acquisition, retention, and transfer: A randomized trial. Physical Therapy, 88(2), 251-260.

Magill, R.A. (2007). Motor learning and control: Concepts and applications ( $8^{\text {th }}$ ed.). New York: McGraw-Hill.

Marchant, D., Clough, P., \& Crawshaw, M. (2007). The effects of attentional focusing strategies on novice dart throwing performance and their task experiences. International Journal of Sport \& Exercise Psychology, 5(3), 291-303.

Marchant, D., Clough, P., Crawshaw, M., \& Levy, A. (2009). Novice motor skill performance and task experience is influenced by attentional focusing instructions and instruction preferences. International Journal of Sport \& Exercise Psychology, 7(4), 488-502.

McNevin, N., Shea, C., \& Wulf, G. (2003). Increasing the distance of an external focus of attention enhances learning. Psychological Research/Psychologische Forschung, 67(1), 22-29.

Perkins-Ceccato, N., Passmore, S., \& Lee, T. (2003). Effects of focus of attention depend on golfers' skill. Journal of Sports Sciences, 21(8), 593.

Poolton, J., Maxwell, J., Masters, R., \& Raab, M. (2006). Benefits of an external focus of attention: Common coding or conscious processing?. Journal of Sports Sciences, 24(1), 89-99. 
Radlo, S., Steinberg, G., Singer, R., Barba, D., \& Melnikov, A. (2002). The influence of an attentional focus strategy on alpha brain wave activity, heart rate, and dartthrowing performance. International Journal of Sport Psychology, 33(2), 205217.

Salmoni, A.W., Schmidt, R.A., \& Walter, C.B. (1984). Knowledge of results and motor learning: A review and critical reappraisal. Psychological Bulletin, 95, 355-386.

Shea, C. \& Wulf, G. (1999). Enhancing motor learning through external-focus instructions and feedback. Human Movement Science, 18(4), 553-571.

Schmidt, R. A. \& Lee, T.D. (2005). Motor Control and Learning: A Behavioral Emphasis ( $4^{\text {th }}$ ed). Champaign, IL: Human Kinetics

Vance, J., Wulf, G., Tollner, T., McNevin, N., \& Mercer, J. (2004). EMG activity as a function of the performer's focus of attention. Journal of Motor Behavior, 36(4), 450-459.

Weiss, S., Reber, A., \& Owen, D. (2008). The locus of focus: The effect of switching from a preferred to a non-preferred focus of attention. Journal of Sports Sciences, 26(10), 1049-1057.

Wulf, G. \& Weigelt, C. (1997). Instructions about physical principles in learning a complex motor skill: To tell or not to tell... Research Quarterly for Exercise and Sport, 68(4), 362-367.

Wulf, G., Prinz, W., \& Hob, M. (1998). Instructions for motor learning: Differential effects of internal versus external focus of attention. Journal of Motor Behavior, 30(2), 169-179. 
Wulf, G., Lauterbach, B., \& Toole, T. (1999). The learning advantages of an external focus of attention in golf. Research Quarterly for Exercise \& Sport, 70(2), 120126.

Wulf, G., McNevin, N., Fuchs, T., Ritter, F., \& Toole, T. (2000). Attentional focus in complex skill learning. Research Quarterly for Exercise \& Sport, 71(3), 229-239.

Wulf, G., McNevin, N., \& Shea, C. (2001). The automaticity of complex motor skill learning as a function of attentional focus. The Quarterly Journal of Experimental Psychology A: Human Experimental Psychology, 54A(4), 1143-1154.

Wulf, G., Shea, C., \& Park, J. (2001). Attention and motor performance: Preferences for and advantages of an external focus. Research Quarterly for Exercise \& Sport, 72(4), 335-344.

Wulf, G., McConnel, N., Gartner, M., \& Schwarz, A. (2002). Enhancing the learning of sport skills through external-focus feedback. Journal of Motor Behavior, 34(2), 171-182.

Zachry, T., Wulf, G., Mercer, J., \& Bezodis, N. (2005). Increased movement accuracy and reduced emg activity as the result of adopting an external focus of attention. Brain Research Bulletin, 67(4), 304-309. 


\section{Appendix A}

\section{Statistical Output}

Results for: Acquisition $3 \times 2 \times 6$ ANOVA

\section{General Linear Model: Score versus Group, Day, Trial Block, Subject}

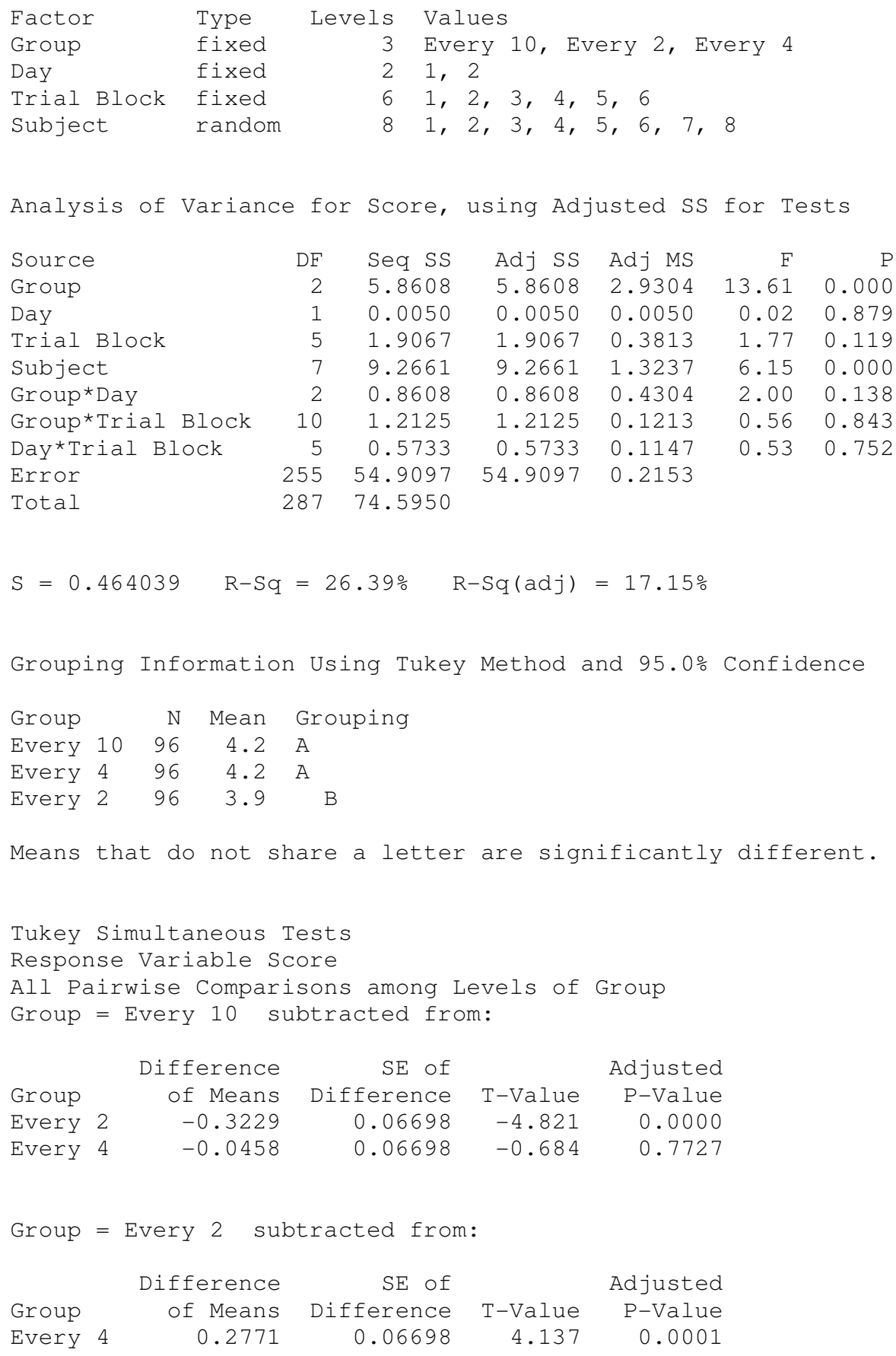




\section{Results for: Retention Test One-Way ANOVA}

\section{One-way ANOVA: Retention versus Group}

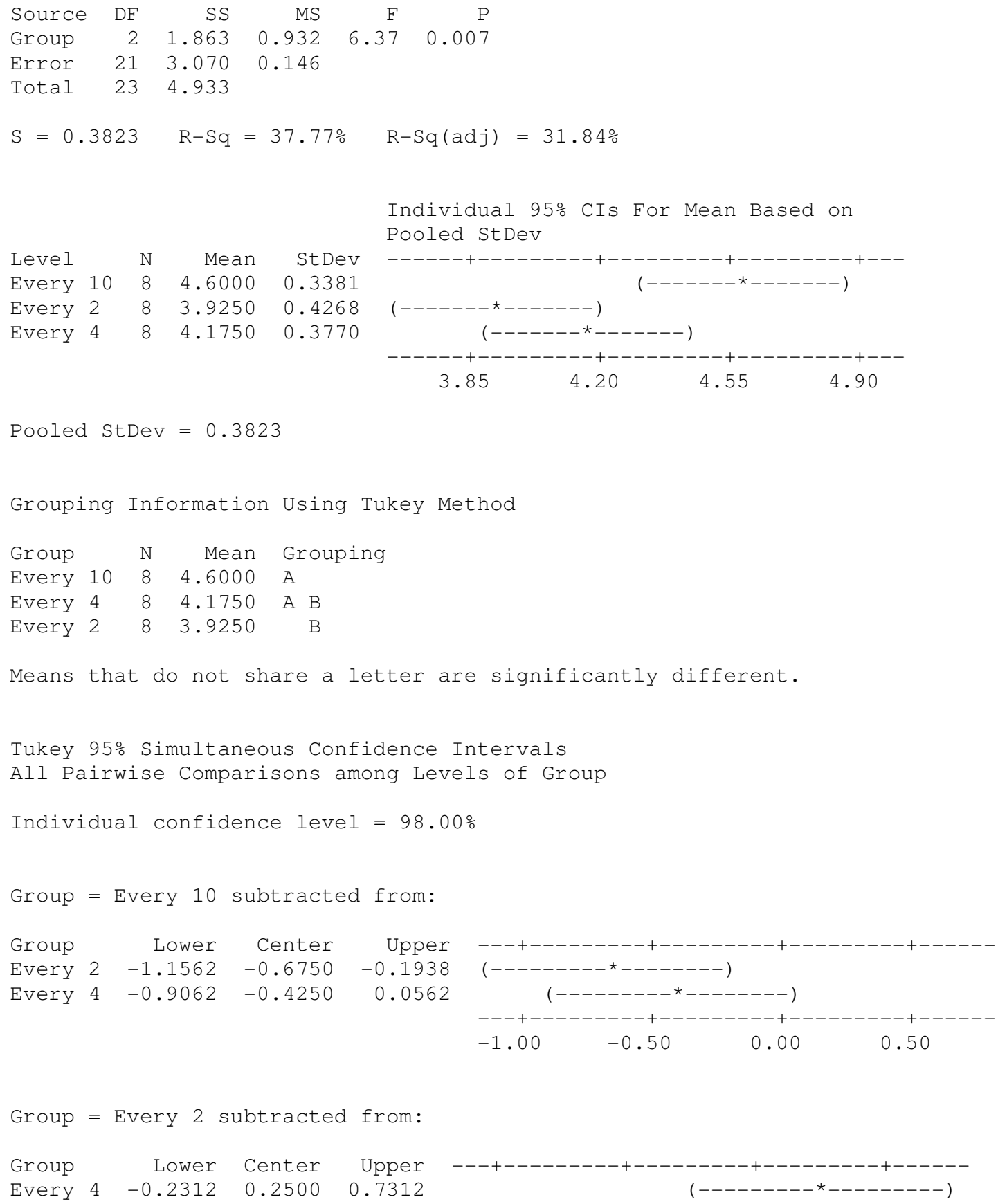


Results for: Transfer Test One-Way ANOVA

\section{One-way ANOVA: Transfer versus Group}

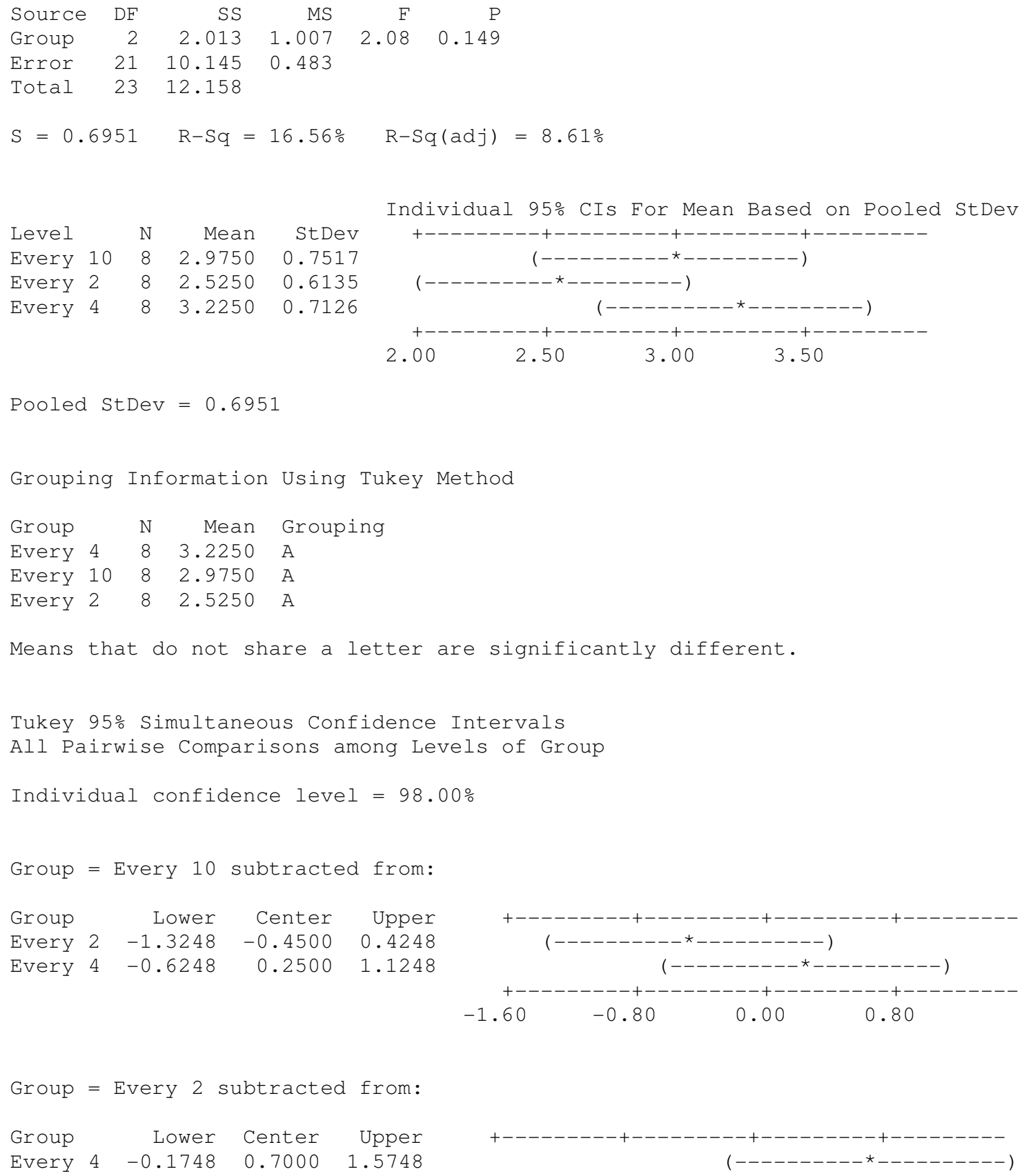




\section{Appendix B}

\section{Informed Consent}

\section{INFORMED CONSENT TO PARTICIPATE IN \\ Effects of Changing Attentional Focus Reminder Rates on Learning a Novel Task}

A research project on attentional focus strategies is being conducted by Arya Alami in the Department of Kinesiology at Cal Poly, San Luis Obispo. The purpose of the study is to examine the effects of differing external focus cue reminder rates on the learning of dart throwing.

You are being asked to take part in this study by learning the technique to correctly throw darts and using an external focus strategy while practicing dart throwing. Your participation will take approximately three hours spread evenly over three days of testing. There will be two consecutive days of practice followed by one day of testing which will be held five days after the second day of practice. Please be aware that you are not required to participate in this research and you may discontinue your participation at any time without penalty.

The possible risks associated with participation in this study include strain or injury to the fingers, wrist or elbow of the throwing arm. If you should experience any finger, wrist or elbow pain associated with dart throwing, please be aware that you may contact Cal Poly Health Services in Building 27 at (805) 756-1211, or the researcher at (925) 212-3477, for assistance.

Your confidentiality will be protected by the researcher. Your name will not appear in reports of this research. Potential benefits associated with this study include learning the proper technique for dart throwing. In addition, your participation may also contribute to the understanding of the acquisition of motor skills.

If you have questions regarding this study or would like to be informed of the results when the study is completed, please feel free to contact Arya Alami at (925) 212-3477 or Dr. Kellie Hall at (805) 756-1786. If you have questions or concerns regarding the manner in which the study is conducted, you may contact Dr. Steve Davis, Chair of the Cal Poly Human Subjects Committee, at (805) 756-2754, sdavis@ @alpoly.edu, or Dr. Susan Opava, Dean of Research and Graduate Programs, at 756-1508, sopava@ calpoly.edu.

If you agree to voluntarily participate in this research project as described, please indicate your agreement by signing below. Please keep one copy of this form for your reference, and thank you for your participation in this research.

Signature of Volunteer

Signature of Researcher

\section{Date}

Date 